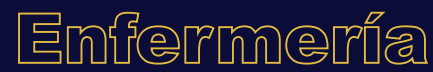

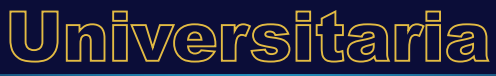

\section{Eventos adversos en recién nacidos hospitalizados en una Unidad de Cuidados Intermedios}

\section{Adverse events among newborns hospitalized in an Intermediate Care Unit}

\section{Eventos adversos em recém-nascidos hospitalizados em una Unidade de Cuidados Intermediários}

\section{Y. Chumpitaz-Chávez ${ }^{\text {a1* }}$, H. Gutiérrez-Crespo ${ }^{\text {b2 }}$, J. Matzumura-Kasano ${ }^{\text {c2 }}$, R.A. Ruiz-Arias ${ }^{\mathrm{d}_{3}}$}

ORCID

${ }^{\mathrm{a}} 0000-0003-1896-7511$

b $0000-0003-1097-6990$

$$
\frac{{ }^{c} \underline{0000-0002-1464-550 X}}{{ }^{\mathrm{d}} \underline{0000-0002-8877-6158}}
$$

${ }^{1}$ Universidad Nacional Mayor de San Marcos, Facultad de Medicina / Instituto Nacional Materno Perinatal, Unidad de Cuidados Intermedios Neonatales, Lima, Perú

${ }^{2}$ Universidad Nacional Mayor de San Marcos, Facultad de Medicina, Lima, Perú

${ }^{3}$ Universidad San Ignacio de Loyola, Facultad de Medicina, Lima, Perú

Recibido: 17 febrero 2020

Aceptado: 2 septiembre 2020

RESUMEN

Introducción: Los recién nacidos (RN) conforman uno de los grupos poblacionales más vulnerables. El riesgo de ocurrencia de un evento adverso durante su hospitalización se incrementa por diversos factores.

Objetivo: Identificar los eventos adversos en recién nacidos hospitalizados en una unidad de cuidados intermedios en 2018. 
Metodología: Estudio de tipo descriptivo, diseño transversal y retrospectivo, realizado en la Unidad de Cuidados Intermedios Neonatales del Instituto Nacional Materno Perinatal durante el 2018. Se elaboró una ficha de recolección de datos que constó de nueve ítems correspondientes a procedimientos realizados por el personal de enfermería. Se utilizó un análisis univariado de los datos, presentados en tablas y gráficos.

Resultados: Fueron analizados 73 eventos adversos, la menor edad gestacional fue de 32 semanas, con un máximo de 28 días de hospitalización. 60\% de eventos se produjo en los RN más lábiles y con situación de salud compleja, de los cuales, 58\% fueron infecciones asociadas a catéter percutáneo o periférico, cuyo germen frecuente fue Sthapylococcus coagulasa negativo. Solo 20.5\% de eventos adversos fueron reportados.

Discusión: Los resultados obtenidos coinciden con otras investigaciones en que los factores intrínsecos de los recién nacidos contribuyen en la aparición de eventos adversos; de igual manera concuerdan en la necesidad de prevenirlos y notificarlos.

Conclusiones: La prematuridad, el tiempo de hospitalización en una unidad crítica y de exposición a diversos procedimientos, incrementaron el riesgo de ocurrencia de eventos adversos en recién nacidos. Detectar oportunamente estos acontecimientos es importante, así como fomentar la cultura de reporte, con el fin de evitarlos.

Palabras clave: Evento adverso; recién nacido; enfermería; Perú.

\section{ABSTRACT}

Introduction: Newborns are one of the most vulnerable populations, and their risk of suffering an adverse event during their hospital stay is influenced by diverse factors.

Objective: To identify the adverse events among newborns hospitalized in an Intermediate Care Unit in 2018.

Methodology: This is a descriptive, transversal, and retrospective study carried out in a Newborn Intermediate Care Unit of the Maternal and Perinatal National Institute during 2018. A data gathering chart with 9 items related to the corresponding nursing procedures was designed. A univariate data analysis was calculated, and the results were shown in tables and graphs.

Results: 73 adverse events were analyzed. The lowest gestational age was 32 weeks, and the longest hospitalization was 28 days. $60 \%$ of these events were related to the more delicate and with the more complex situation newborns; and $58 \%$ of these were related to percutaneous or peripheral catheter infections and the most frequent pathogen was Coagulase-Negative Staphylococcus. Only $28.5 \%$ of the events were reported.

Discussion: These findings are in line with the results of other research studies. Addressing the diverse factors which contribute to the adverse events in newborns in Intermediate Care Units is suggested.

Conclusions: Being premature and the length of the hospital stay increase the risk of adverse events in newborns. It is critical to promote a culture of events reporting.

Keywords: Adverse event; newborn; nursing; Peru.

\section{RESUMO}

Introdução: Os recém-nascidos (RN) constituem um dos grupos populacionais mais vulneráveis. O risco de ocorrência de um evento adverso durante sua hospitalização é incrementado por diversos fatores. 
Objetivo: Identificar os eventos adversos em recém-nascidos hospitalizados em una unidade de cuidados intermediários em 2018.

Metodologia: Estudo de tipo descritivo, desenho transversal e retrospectivo, realizado na Unidade de Cuidados Intermediários Neonatais do Instituto Nacional Materno Perinatal durante 2018. Foi elaborada uma ficha de coleta de dados que constou de nove itens correspondentes a procedimentos realizados pelo pessoal de enfermagem. Utilizou-se uma análise univariáda dos dados, apresentados em tabelas e gráficos.

Resultados: Foram analisados 73 eventos adversos, a menor idade gestacional foi de 32 semanas, com máximo de 28 dias de internação. 60\% dos eventos produziram-se nos RN mais lábeis e em situação de saúde complexa, dos quais, 58\% foram infecções associadas a cateter percutâneo ou periférico, cujo germe frequente foi o Sthapylococcus coagulase negativo. Apenas $20.5 \%$ de eventos adversos foram relatados.

Discussão: Os resultados obtidos coincidem com outras pesquisas em que os fatores intrínsecos dos recém-nascidos contribuem no aparecimento de eventos adversos; aliás, concordam com a necessidade de preveni-los e notificá-los.

Conclusões: A prematuridade, o tempo de hospitalização em uma unidade crítica e de exposição a diversos procedimentos, incrementaram o risco de ocorrência de eventos adversos em recém-nascidos. É importante detectar estes acontecimentos oportunamente, assim como fomentar a cultura de notificação, a fim de evitá-los.

Palavras chave: Evento adverso; recém-nascido; enfermagem; Peru.

\section{INTRODUCCIÓN}

A lo largo de los años, la seguridad en la atención sanitaria continúa siendo un desafío importante a nivel nacional e internacional ${ }^{1}$. El cuidado de la salud ha evolucionado notablemente en diversos aspectos, incluido el ámbito tecnológico que, si bien es cierto, ha logrado reducir significativamente la morbimortalidad de la población en general, trae consigo un incremento en el riesgo de aparición de ciertas complicaciones². Según diversos estudios, una de las poblaciones más vulnerables son los recién nacidos, debido a que, por sus propias características físicas y fisiológicas como la edad gestacional y peso, requieren una atención multidisciplinaria de mayor complejidad y una mayor estancia hospitalaria ${ }^{3-5}$.

Como resultado de la atención sanitaria del recién nacido y con el fin de proveer un buen cuidado al paciente, se pueden generar diversos incidentes conocidos como eventos adversos, los cuales se definen como "[...] aquel(los) que generan daño al paciente, causado(s) después de que éste ingresa a la institución médica y está relacionado más con el cuidado proveído o con ocasión de éste que con la enfermedad de base"6.

Dichos eventos son producidos por múltiples factores, sean intrínsecos (características del paciente), extrínsecos (tratamiento y cuidados hospitalarios) o del sistema institucional (recurso humano y errores de procesos)7. Asimismo, su impacto afecta al recién nacido, causándole desde daños leves hasta severos (en diferentes niveles como neurológico, sensorial, motor o cognitivo); a la familia le genera estrés, preocupación, ansiedad, entre otros, de modo que influye negativamente en la dinámica familiar;; y a la institución, ya que implica, principalmente, un incremento de costos por estancia hospitalaria prolongada e incremento de morbimortalidad9. Cabe mencionar que, dentro del equipo multidisciplinario, es el profesional de enfermería quien realiza la 
mayor cantidad de procedimientos invasivos, los cuales ponen en riesgo al paciente de padecer algún evento, que, según múltiples estudios son considerados $75 \%$ evitables ${ }^{10}$.

A pesar del gran porcentaje de evitabilidad de algunos eventos adversos, continúan siendo un gran problema que afecta la salud de los pacientes. Esto fue revelado por un estudio multicéntrico titulado Seguridad de los pacientes en hospitales de Latinoamérica, en el que se evaluaron diferentes servicios incluido el área de neonatología, donde se reportaron cifras de incidencia de eventos adversos a nivel mundial del 16.6\% en Australia, 12.9\% en Nueva Zelanda, 7.5\% en Canadá, 9.3\% en España, 5.7\% en Holanda, 12.3\% en Suecia, 3.7\% en Estados Unidos y en Latinoamérica, 9.2\%, de los cuales, el $43.5 \%$ es evitable ${ }^{11}$.

En el Perú existen pocos estudios sobre la prevalencia de eventos adversos en la población neonatal, pero una investigación realizada en 2009 demostró que la prevalencia de dichos eventos fue del $11.6 \%$, de los cuales $39.6 \%$ estuvo relacionado con las infecciones asociadas a la atención en salud, $28.9 \%$ con procedimientos, $10.8 \%$ con diagnósticos, $8.4 \%$ con la medicación, $6.9 \%$ a los cuidados y $3.5 \%$ a otros eventos; sin embargo, en Pediatría la prevalencia fue de $10.2 \%, 58.8 \%$ era evitable ${ }^{12}$.

Debido a la problemática antes mencionada, las instituciones de salud han implementado diversas políticas con la finalidad de prevenir los eventos adversos, tal es el caso de los denominados sistemas de registro y notificación de eventos adversos en instituciones de salud ${ }^{13}$. No obstante, muchos profesionales, al desconocer que estos no tienen carácter punitivo, dan lugar a subregistros o que no se reporten los eventos, a pesar de que existen disposiciones normativas precisas ${ }^{14,15}$. La importancia del uso de sistemas de registros es planteada en una investigación basada en la revisión de historias clínicas neonatales, la que, además de brindar información precisa sobre los eventos presentados, contribuyó a desarrollar una herramienta metodológica capaz de alertar sobre el riesgo de ocurrencia de dichos eventos³.

Existen pocas investigaciones sobre la incidencia de eventos adversos en relación con la atención sanitaria neonatal; es por ello que el objetivo del presente estudio plantea identificar los eventos adversos en recién nacidos hospitalizados en la Unidad de Cuidados Intermedios Neonatales (UCIN) del Instituto Nacional Materno Perinatal de Lima, Perú, en el 2018. Esto con la finalidad de brindar una atención segura y de calidad al paciente, proporcionar alternativas que contribuyan a prevenir dichos eventos o sus posibles complicaciones en el recién nacido, así como fomentar una cultura de reporte de eventos en los distintos profesionales de la salud.

\section{METODOLOGÍA}

El presente estudio es de tipo descriptivo, de diseño transversal y retrospectivo. La población estuvo constituida por 100 recién nacidos que estuvieron hospitalizados durante 2018 en la UCIN del Instituto Nacional Materno Perinatal de Lima, Perú, y que presentaron eventos adversos. Esta unidad está conformada por seis áreas: IA (pacientes complejos que requieren oxigenoterapia en fase I o II, calor y monitoreo permanente, prematuros en su mayoría, con diversas malformaciones); IB (pacientes que requieren oxígeno en fase I, con leve dificultad respiratoria, en su mayoría crónicos, y mayores de 37 semanas de edad gestacional, con peso mayor a 2 kilogramos); II (prematuros normotérmicos, para recuperación nutricional); III (pacientes normotérmicos con peso entre 1750gr y 2500 gr, con malformaciones correspondientes a neurocirugía, pero que no dependen de oxígeno); IV (pacientes normotérmicos, con peso mayor a 250ogr, que reciben tratamiento de fototerapia o antibioticoterapia), y Cirugía Neonatal (pacientes normotérmicos en fase pre o posoperatoria, 
que no requieren apoyo de oxígeno). Cabe mencionar que el total de la población corresponde al promedio aproximado de eventos adversos según la estadística anual.

Se empleó un muestreo no probabilístico censal, la muestra la constituyeron los datos de 73 recién nacidos, hospitalizados entre enero y diciembre del 2018, quienes presentaron por lo menos un evento adverso durante ese periodo. Dentro de los criterios de inclusión se consideraron las fichas de eventos adversos notificadas por el personal de enfermería que tenía un mínimo de diez meses de antigüedad laboral, de pacientes con edad igual o menor a 28 días de vida que estuvieron hospitalizados en la UCIN en el periodo delimitado. Fueron excluidas aquellas fichas incorrectamente llenadas o reportadas por otros miembros del equipo de salud.

Los eventos adversos fueron clasificados como leves (presencia de daños mínimos, no es necesaria la intervención o es mínima sin prolongar la estancia), moderados (daño o pérdida funcional permanente o de larga duración que prolonga la estancia hospitalaria y requiere de cirugía o un tratamiento suplementario) y graves (reduce la esperanza de vida o causa pérdida funcional importante, permanente o de larga duración, requiere de una intervención quirúrgica o médica mayor) ${ }^{16}$.

Se utilizaron como fuentes de información las historias clínicas y los registros de eventos adversos reportados a la oficina de calidad, en el periodo deintervención delainvestigación, cuyos datos fueron plasmados en una ficha de recolección, la cual constituyela unidad de análisis. Para reducir el sesgo de información, el instrumento fue elaborado considerando algunos factores relacionados a eventos adversos, y estuvo conformado por nueve ítems, agrupados en tres dimensiones: eventos adversos asociados a la medicación, al cuidado de dispositivos invasivos, y no invasivos. Asimismo, fue sometido a un análisis de validez de contenido por seis jueces expertos, mediante el índice de Kappa, cuyo resultado fue $\mathrm{K}=0.855$, y de confiabilidad, utilizando Kuder-Richardson 20, con el cual se obtuvo un resultado de 0.71 .

El análisis de datos fue desarrollado en dos partes, la primera recopiló las características sociodemográficas de los recién nacidos (edad gestacional, sexo, días y área de hospitalización), mientras que el segundo segmento correspondió a los eventos adversos (clasificados según sus tres dimensiones [asociadas a medicación, dispositivos invasivos y no invasivos], gravedad del evento y reporte por el personal de enfermería).

A su vez, para el análisis de los datos se realizó un análisis univariado mediante un estudio descriptivo de los datos, presentados en tablas y gráficos de acuerdo con las variables. La información obtenida fue ingresada en el programa Excel 2016 y analizada mediante el software IBM SPSS v.24.o. Cabe recalcar que en esta investigación sólo se analizaron los eventos adversos relacionados con el cuidado de enfermería.

El presente trabajo fue evaluadoy aprobado por el Comité de Ética e Investigación de lainstitución de salud, posterior a ello se procedió a la recolección de los datos y la utilización de registros de los eventos adversos. Durante ese proceso se mantuvieron los principios de confidencialidad y anonimato de los participantes; asimismo, la investigación no representó ningún riesgo para los recién nacidos, pues sólo se trabajó con información ya registrada sobre ellos.

\section{RESULTADOS}

En la presente investigación se analizaron los datos de un total de 73 recién nacidos que presentaron eventos adversos durante su hospitalización en alguna de las seis áreas que conforman la UCIN. Con respecto a las características sociodemográficas, se reveló que 54.7\% fueron recién 
nacidos pretérmino, cuya edad gestacional promedio fue 36.2 semanas. En relación con los días de hospitalización se encontró que el menor tiempo de estancia hospitalaria fue de dos días, y el mayor de 28 días, con un promedio de 16.5 días.

Del total de recién nacidos se encontró que 60.3\% fueron atendidos en el área de Intermedios IA, de los cuales, 26 eran de sexo femenino, mientras que 18 del masculino. Por otro lado, en el área de Cirugía Neonatal se obtuvo una proporción de 2 a 1 predominando el sexo masculino (Tabla 1).

Respecto a los componentes de las dimensiones de los eventos adversos se encontró que $57.8 \%$ de estos fueron infecciones asociadas al uso de catéter percutáneo o periférico (PICC o CVP), de los cuales $75 \%$ presentó hemocultivos positivos para el germen Sthapylococcus coagulasa negativo y $14.5 \%$ para Acinetobacter. Los casos reportados de flebitis ocuparon el segundo lugar, puesto que se presentaron en $12.1 \%$ del total. No se encontraron eventos adversos asociados al medicamento ni a frecuencia errónea; sin embargo, se encontró un evento relacionado al modo de administración incorrecta (Tabla 2).

El estudio mostró que, según la gravedad de los eventos adversos, 65.8\% de recién nacidos hospitalizados presentaron eventos moderados, a diferencia de los eventos leves, cuyo valor fue de $34.2 \%$. Cabe destacar que no hubo eventos graves.

Tabla 1. Descripción del grupo de estudio según área de
hospitalización y sexo

Tabla 2. Clasificación de eventos adversos según componentes de cada dimensión

\begin{tabular}{lrr}
\hline Dimensiones & $\mathrm{n}$ & $\%$ \\
\hline Medicamentos & 0 & 0 \\
Fecuencia errónea & 0 & 0 \\
Medicamento erróneo & 1 & 1.2 \\
Modo de administración incorrecto & & \\
$\begin{array}{l}\text { Dispositivos Invasivos } \\
\text { Infecciones asociadas a catéter percutáneo o }\end{array}$ & 48 & 57.8 \\
periférico & & \\
Desplazamiento, ruptura u obstrucción de & 4 & 4.8 \\
catéter venoso central o periférico & 10 & 12.1 \\
Flebitis & 11 & 13.3 \\
Extravasación medicamentosa & & \\
Obstrucción de catéter umbilical arterial o & 1 & 1.2 \\
venoso & & \\
Dispositivos no invasivos & & \\
Lesión de columnela nasal secundaria a & 8 & 9.6 \\
\hline dispositivo de oxigeno (cánula binasal o CPAP) & &
\end{tabular}


La relación de la gravedad en el total de eventos adversos en recién nacidos hospitalizados en la UCIN, de acuerdo al área de hospitalización, se muestra en la Figura 1. En el área de Intermedios IA se produjeron un total de 20.5\% de eventos adversos leves y $39.7 \%$ de eventos moderados. En el área de Intermedios III los eventos adversos leves constituyeron $2.7 \%$, a diferencia de los eventos moderados que fueron 9.6\%. En el área de Cirugía Neonatal el porcentaje de eventos leves fue mayor al de moderados (2.7\% vs $1.4 \%)$.

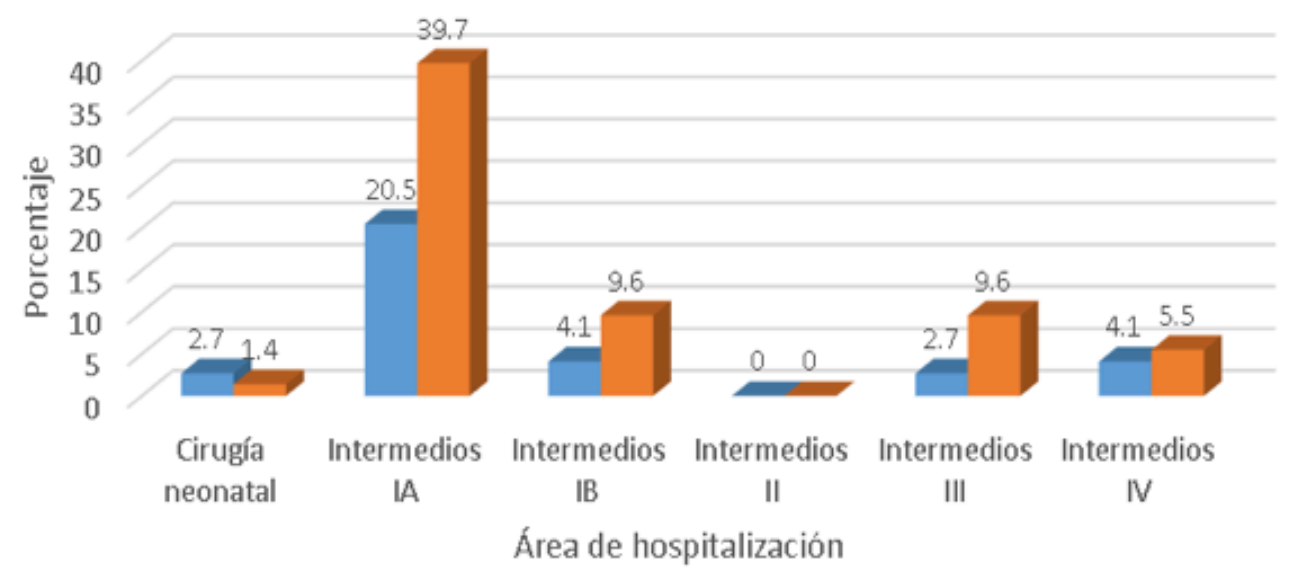

Eventos adversos leves Eventos adversos moderados

Figura 1. Clasificación de eventos adversos según su gravedad y el área de hospitalización

En lo que respecta al reporte del evento por el personal de enfermería, se obtuvo que en el total de eventos adversos moderados no hubo reporte, y en los eventos adversos calificados como leves, en 15 de 25 este se realizó.

\section{DISCUSIÓN}

Los eventos adversos son considerados como daños leves, moderados e incluso severos. Estos se pueden producir en el paciente como consecuencia de la atención sanitaria brindada. La cantidad de eventos adversos va en constante aumento, principalmente en poblaciones vulnerables como los recién nacidos, quienes por sus características sociodemográficas, presentan un mayor riesgo de ocurrencia de dichos eventos. Esto ocurre con la prematuridad, pues a menor edad gestacional, los tiempos de hospitalización y de exposición a procedimientos invasivos son mayores y, durante el internamiento, se producen entre uno y tres incidentes en el paciente ${ }^{17,18}$.

En la presente investigación se encontró que la edad gestacional promedio fue de 36 semanas, lo cual corresponde a un recién nacido pretérmino ${ }^{19}$. Este tipo de nacimiento está condicionado por el aumento de sobrevida de los pacientes nacidos con muy bajo peso y de extremo bajo peso, así como la presencia de malformaciones, las cuales agravan el estado del paciente, pues también afectan su neurodesarrollo ${ }^{20,21}$. Otra característica relevante es que los recién nacidos de sexo masculino presentan asociación significativa con el resultado adverso; es decir, tienen mayor riesgo de enfermar a diferencia del sexo femenino ${ }^{22}$, lo que difiere con esta investigación. 
En la actualidad, la mayoría de las unidades que brindan cuidados neonatales especializados albergan recién nacidos de alto riesgo, quienes se encuentran expuestos a múltiples y complejos tratamientos ${ }^{23}$, diversos procedimientos, infinidad de medicaciones y constantes manipulaciones, cuyo exceso resulta nocivo para el paciente, independientemente del personal de salud que lo realiza ${ }^{24}$.

En relación con el área de hospitalización de los recién nacidos que presentaron algún evento adverso, se encontró que en el área de Intermedios IA se obtuvo el mayor porcentaje de eventos ya sea moderados o leves, seguido por Intermedios IB e Intermedios III y IV. Esto puede deberse a diversos factores, como la diferencia en los criterios de ingreso de los pacientes, a la complejidad de atención que requiereny a lafalta de personal de enfermería para satisfacer todas sus necesidades. Cabe mencionar que un paciente altamente complejo demanda atención y cuidados permanentes, lo que se traduce en mayor carga laboral y afecta no sólo la calidad del cuidado prestado a otros pacientes, sino también incrementa el riesgo de ocurrencia de eventos adversos 25 .

Los errores de tratamiento son una de las causas frecuentes de aparición de eventos adversos ${ }^{26}$. Tales eventos se producen debido a factores internos, externos y relacionados con el sistema, de los cuales, los últimos tienen mayor predominio ${ }^{10}$. De las dimensiones analizadas en este estudio, la mayoría fueron infecciones asociadas a catéteres endovenosos, percutáneos o periféricos, lo cual concuerda con algunas investigaciones ${ }^{10,11,27}$. Dichas infecciones son las que tienen mayor impacto en la morbimortalidad de los pacientes hospitalizados, ya que uno de los procedimientos realizados con mayor frecuencia en la unidad neonatal es la cateterización de accesos venosos. Si bien este procedimiento implica grandes ventajas a favor del paciente, paradójicamente, el material del cual está fabricado es frágil y requiere cuidados específicos para su mantenimiento. Por ejemplo, su manipulación provoca, en algunos casos, obstrucción parcial o total del catéter y, en otros, ruptura del mismo. Así, la frecuente manipulación de dicho catéter es un factor de riesgo para contraer infecciones ${ }^{28,29}$ y generar diversos cuadros clínicos que agravan el estado de salud ${ }^{30}$, pues en este grupo poblacional específico la incidencia de infección puede alcanzar $25 \%$ o más ${ }^{31}$.

En adición, a nivel mundial los Staphylococcus aureus son los agentes etiológicos principales ${ }^{32}$ al ser gérmenes habituales de la piel, los cuales pueden causar infección si hay colonización en el sitio de inserción, ya que los microorganismos pueden acceder por capilaridad a través del túnel dérmico que queda alrededor del catéter hasta alcanzar la punta (vía más frecuente). En el caso de catéteres de larga duración, 10-50\% de los casos ocasionan infección, puesto que existe mayor contaminación de los lúmenes de los conectores por la manipulación frecuente de los mismos 33 .

Respecto a la clasificación de eventos adversos, en este caso, en su gran mayoría fueron de tipo moderado, lo que significa que se prolongó la estancia hospitalaria ${ }^{12}$. Es importante mencionar que las características del propio paciente, además de la presencia de algún evento adverso, incrementan los riesgos potenciales a corto y largo plazo, debido a las complicaciones y la hospitalización prolongada, a diferencia de otro paciente que no presentó ningún evento34. Asimismo, la tasa general de eventos adversos en el Perú varía en contraste con la de los países desarrollados, quienes cuentan con mejores condiciones sanitarias que disminuirían, de cierto modo, el riesgo de que se presente algún evento inesperado 35 .

A pesar de las políticas y programas implementados por las instituciones de salud, aún no se consiguen mejoras notables respecto al reporte de los eventos adversos, lo cual coincide con los resultados de esta investigación. En efecto, 79.5\% del total de enfermeras no reportó la ocurrencia 
de dichos acontecimientos. Una de las causas del no reporte es el temor a la medida punitiva, que depende de la magnitud e impacto del evento ${ }^{14,15}$. Si bien es cierto que $75 \%$ de eventos son evitables ${ }^{10}$, en su mayoría no son reportados a pesar de que existe un sistema de registro y notificación de eventos adversos en instituciones de salud, así como disposiciones normativas precisas que lo avalan con el fin de proveer un mejor cuidado ${ }^{13}$. En un estudio reciente los investigadores comprobaron que hay un vacío de investigaciones sobre la problemática antes mencionada, en especial de los eventos adversos registrados en las unidades de cuidados críticos, ya sea de cuidados intermedios o intensivos neonatales ${ }^{36}$.

Las limitaciones que se suscitaron al realizar la presente investigación fueron la falta de sistematización de registros de reporte de eventos adversos, lo cual generó que se realizaran subregistros o, en su defecto, que algunos casos no fueran incluidos. Es importante emplear definiciones estandarizadas de eventos adversos, pues es transcendental para la homogeneidad de los datos ${ }^{37}$. A su vez, se evidenció que la cultura de reporte de eventos fue escasa.

Por otro lado, las limitaciones propias del tipo de muestreo utilizado pudieron generar sesgo debido a la falta de representatividad de la muestra, ya que no permitieron que se realizaran inferencias a otros grupos poblacionales ni a otras unidades neonatales, pues existen pocas investigaciones similares que permitan efectuar procedimientos, en su mayoría invasivos, y comparaciones de prevalencia de dichos eventos.

\section{CONCLUSIONES}

Los eventos adversos producidos en recién nacidos de alto riesgo son influenciados por factores como la prematuridad, la cual implicó un mayor tiempo de hospitalización en unidades neonatales críticas y de exposición a diversos procedimientos que incrementaron el riesgo de infección. En pacientes con larga estancia hospitalaria se presentó por lo menos un evento adverso. Además, prevalecieron las infecciones asociadas a catéteres percutáneos o periféricos, cuyo germen predominante fue el Staphylococcus coagulasa negativo, que los convirtió en eventos adversos moderados y en consecuencia, pudieron agravar el estado de salud del recién nacido. Finalmente, una cantidad considerable de estos eventos no fueron reportados, lo cual generó un impacto negativo para el paciente, familia e institución de salud.

Con base en lo anterior, la presente investigación sirve como marco de referencia para considerar nuevos enfoques que permitan comprender el impacto de la atención de enfermería en el paciente neonato, a nivel nacional e internacional, así como la asociación con otras variables (carga laboral, clima laboral, aplicación de protocolos, cultura de seguridad, cultura de reporte de eventos adversos, entre otras) que puedan comprometer el estado de salud del paciente.

\section{RESPONSABILIDADES ÉTICAS}

Protección de personas y animales. Los autores declaran que para la presente investigación no se realizaron experimentos en personas ni animales.

Confidencialidad. En la presente investigación no se exponen datos que revelen la identidad de los pacientes.

Conflicto de intereses. Los autores declaran no tener conflicto de intereses.

Financiamiento. Ninguno.

Agradecimientos. A la Oficina de Calidad y Jefaturas de la Unidad de Cuidados Intermedios Neonatales del Instituto Nacional Materno Perinatal. 


\section{REFERENCIAS}

1. Podestá-Gavilano LE, Maceda-Kuljich M. Calidad percibida del servicio y cultura de seguridad en salud en el personal médico del Hospital II EsSalud Vitarte. Lima, 2017. Horiz. Med. 2018; 18(3): 48-56. https://dx.doi.org/10.24265/horizmed.2018.v18n3.08

2. Torres-Almeida CM, Nuno Alves-dos Santos Almeida F, Jacinto-Escola JJ, Costa Pereira-Rodrigues VM. A influência tecnológica no cuidar dos profissionais de saúde: tradução e adaptação de escalas. Rev. Latino-Am. Enfermagem. 2016; 24(e2681): 1-9.

https://dx.doi.org/10.1590/1518-8345.0990.2681

3. Sánchez-Bañuelos LG, Pérez-Gutiérrez J, Tamariz-Velázquez FE, Delgado-Rubio M. Seguridad del neonato hospitalizado. Aproximaciones y propuestas. Enferm. univ. 2012; 9(9): 27-36.

https://doi.org/10.22201/eneo.23958421e.2012.2.235

4. Ticona-Rendon M, Huanco-Apaza D, Ticona-Huanco D. Incidencia, supervivencia y factores de riesgo del recién nacido con extremo bajo peso en el hospital. Acta méd. peruana. 2015; 32(4): 211-20. https://bit.ly/3azRdR2

5. Schwoebel A, Creely J. Improving the safety of neonatal care through the development and implementation of a staff-focused delta team. J Perinatal Neonatal Nurs. 2010; 24(1): 81-7.

https://dx.doi.org/10.1097/JPN.obo13e3181се3заb

6. Bautista-Rodríguez LM, Parada-Rico DA, Ballesteros N, Rodríguez J, Ornella-Carreño Z, GuampeBayona L, et al. Frecuencia, evitabilidad y consecuencias de los eventos adversos. rev. cienc. cuidad. 2011; 8(1): 75-83. https://bit.ly/3qDuHw1

7. Parellada-Blanco J, Hidalgo-Sánchez AO, Cruz LR, González-Corrales Y. Eventos adversos en cuidados intensivos. Rev Cub Med Int Emerg. 2018; 17(3): 1-16. https://bit.ly/3s8Afir

8. Acosta MF, Cabrera-Bravo N. Percepción de padres de hijos prematuros frente a la hospitalización en la Unidad de Cuidado Intensivo Neonatal: un estudio de revisión documental. Revista UNIMAR. 2016; 34(1), 193-9. https://bit.ly/2ZzGoYA

9. Martínez-Valverde S, Castro-Ríos A, Salinas-Escudero G, Villasis-Keever MA, Garduño-Espinosa J, Muñoz-Hernández O. Direct medical costs of neonatal respiratory distress syndrome in two specialized public hospitals in Mexico. Salud pública Méx 2014; 56(6): 612-8.

https://doi.org/10.21149/spm.v56i6.7387

10. Zárate-Grajales R, Olvera-Arreola S, Hernández-Cantoral A, Hernández-Corral S, Sánchez-Angeles $S$, Valdez-Labastida R, et al. Factores relacionados con eventos adversos reportados por enfermería en unidades de cuidados intensivos. Proyecto multicéntrico. Enferm. univ. 2015; 12 (2): 63-72. http://dx.doi.org/10.1016/j.reu.2015.03.003

11. Ministerio de Sanidad/Organización Mundial de la Salud. Estudio IBEAS: Prevalencia de efectos adversos en Hospitales de Latinoamérica. Madrid: Ministerio de Sanidad/OMS; 2010.

12. Organización Mundial de la Salud/Ministerio de Salud. Estudio Iberoamericano de Efectos Adversos ligados a la Hospitalización en el Perú. IBEAS PERÚ. Lima: OMS/Ministerio de Salud; 2007.

13. Vallejo-Gutiérrez P, Bañeres-Amella E, Sierra E, Casal J, Agra Y. Lessons learnt from the development of the Patient Safety Incidents Reporting a Learning System for the Spanish National Health System: SiNASP. Rev Calid Asist. 2014; 29(2): 69-77.

https://dx.doi.org/10.1016/j.cali.2013.09.007

14. Marques da Silva-de Paiva MC, Popim RC, Melleiro MM, Rizatto-Tronchim DM, Molina-Lima SA, Casquel Monti-Juliani CM. The reasons of the nursing staff to notify adverse events. Rev. LatinoAm. Enfermagem. 2014; 22(5): 747-54. https://dx.doi.org/10.1590/0104-1169.3556.2476 
15. Burbano-Valdés HM, Caicedo-Eraso ME, Cerón-Burgos A, Jacho-Caicedo C, Yépez-Chaparro MC. Causas del no reporte de eventos adversos en una Institución Prestadora de Servicios de Salud en Pasto-Nariño, Colombia. Rev Univ. Salud. 2013; 15(2): 187-95.

16. Organización mundial de la Salud. Marco Conceptual de la Clasificación Internacional para la Seguridad del Paciente. En: Organización Mundial de la Salud. Marco Conceptual de la clasificación Internacional para la Seguridad del Paciente Versión 1.1. Ginebra: OMS; 2009.

17. Subhedar NV, Parry HA. Critical incident reporting in neonatal practice. Arch Dis Child Fetal Neonatal Ed. 2010; 95(5): 378-82. https://doi.org/10.1136/adc.2008.137869

18. Da Silva-Lanzillotti L, Horsth-De Seta M, Tavares-de Andrade CL, Vieira-Mendes Junior W. Adverse events and other incidents in neonatal intensive care units. Cien Saude Colet. 2015; 20(3): 937-46. http://dx.doi.org/10.1590/1413-81232015203.16912013

19. Gómez-Gómez M, Danglot-Banck C, Aceves-Gómez M. Clasificación de los niños recién nacidos. Rev Mex Pediatr. 2012; 79(1): 32-9.

20. Blencowe H, Lee ACC, Cousens S, Bahalim A, Narwal R, Zhong N, et al. Preterm birth-associated neurodevelopmental impairment estimates at regional and global levels for 2010. Pediatr Res. 2013; 74(Suppl 1): 17-34. https://doi.org/10.1038/pr.2013.204

21. Fernández-Sierra C, Matzumura-Kasano J, Gutiérrez-Crespo H, Zamudio-Eslava L, Melgarejo-García G. Secuelas del neurodesarrollo de recién nacidos prematuros de extremadamente bajo peso y de muy bajo peso a los dos años de edad, egresados de la Unidad de Cuidados Intensivos Neonatales del Hospital Nacional Edgardo Rebagliati Martins 2009-2014. Horiz. Med. 2017; 17(2): 6-13. https://doi.org/10.24265/horizmed.2017.v17n2.01

22. Peelen MJ, Kazemier BM, Ravelli AC, De Groot CJM, Van Der-Post JA, Mol BWJ, et al. Impact of fetal gender on the risk of preterm birth, a national cohort study. Acta Obstet Gynecol Scand. 2016; 95(9): 1034-41. https://doi.org/10.1111/aogs.12929

23. Ceriani-Cernadas J. La morbilidad reemplaza a la mortalidad: un dilema ético en el cuidado de los prematuros en los límites de la viabilidad. Arch Argent Pediatr. 2012; 110(2): 98-9. https://dx.doi.org/10.5546/aap.2012.98

24. Rodríguez-González L, De la Mata-Alcoba I. Procedimiento de mínima manipulación en el neonato. Rev. enferm. CyL. 2014; 6(2): 58-64. https://bit.ly/3dvt5AE

25. Romero-Massa E, Lorduy-Bolívar JP, Pájaro-Melgar C, Pérez-Duque, CA. Relación entre la carga laboral de enfermería y la gravedad del paciente en unidades de cuidado intensivo de adultos. Aquichan. 2011; 11(2): 173-86. https://doi.org/10.5294/aqui.2011.11.2.4

26. Esqué-Ruiz MT, Moretones-Suñol MG, Rodríguez-Miguélez JM, Sánchez-Ortiz E, Izco-Urroz M, de Lamo-Camino $M$, et al. Los errores de tratamiento en una unidad neonatal, uno de los principales acontecimientos adversos. An. pediatr. 2016; 84(4): 211-7.

https://doi.org/10.1016/j.anpedi.2015.09.009

27. Alves-Moreira I, Queiroz-Bezerra AL, Tanferri de Brito-Paranaguá T, Bauer de Camargo-Silva AE, Machado de Azevedo-Filho F. Conhecimento dos profissionais de saúde sobre eventos adversos em unidade de terapia intensiva. Rev enferm UERJ. 2015; 23(4): 461-7.

http://dx.doi.org/10.12957/reuerj.2015.5158

28. Padilla-Sánchez C, Montejano-Lozoya R, Benavent-Taengua L, Monedero-Valero A, Borras-Vañó MJ, Ángel-Selfa MJ, Riera-Torres MJ. Risk factors associated with adverse events in neonates with peripherally inserted central catheter. Enferm Intensiva. 2019. 30(4): 170-80.

https://doi.org/10.1016/j.enfi.2018.10.006 
29. Álvarez-Hernández G, Amaro-Ortega C. Costos atribuibles y factores de riesgo de infección nosocomial en un hospital pediátrico del Estado de Sonora, 2008. Bol. Med. Hosp. Infant. Mex. 2010; 67(2): 118-27. https://bit.ly/3qBoMaY

30. Pérez-Camacho P, Pino-Escobar J, Cleves-Luna D, Torres-Mosquera A, Rosso-Suarez F, BallesterosCastro A. Características clínicas y paraclínicas de recién nacidos con sepsis en un hospital nivel IV en Cali, Colombia. Infect. 2018; 22(3): 141-6. http://dx.doi.org/10.22354/in.v22i3.725

31. Robaina-Castellanos GR, Riesgo-Rodríguez SC. Sepsis neonatal y neurodesarrollo en recién nacidos de muy bajo peso en Matanzas, Cuba 2006-2010: cohorte prospectiva. Medwave. 2016;16(3): e6422. https://doi.org/10.5867/medwave.2016.03.6422

32. Ulloa-Ricárdez A, Salazar-Espino B. Epidemiología de infección neonatal temprana y tardía en una Unidad de Cuidados Intensivos Neonatales. Rev Hosp Jua Mex. 2019; 86(3): 110-5. https://bit.ly/zudDPK1

33. García-Rodríguez J, de Pablos-Gómez M, Gutiérrez-Altés A. El microbiólogo y la infección asociada a catéter. Rev Esp Quimioter 2010; 23(2): 53-62. https://bit.ly/3axFmCR

34. Spironello RA, Nakamura-Cuman RK. Caracterização de eventos adversos em uma unidade de terapia intensiva neonatal. Rev. Recien. 2019; 9(28):131-6. https://bit.ly/3s8fkfu

35. Espíritu N, Lavado G, Pantoja L, Lam C, Barrientos M, Centeno R. Notificación de eventos adversos en hospital nacional en Lima. Rev. calid. asist. 2007; 22(6): 335-41.

https://doi.org/10.1016/S1134-282X(07)71242-3

36. Parra-Pareja CV, López-Rivas JS, Puerto-Guerrero AH, Galeano-Goyes ML. Eventos adversos en un hospital pediátrico de tercer nivel de Bogotá. Rev. Fac. Nac. Salud Pública. 2017; 35(2): 284-92. https://doi.org/10.17533/udea.rfnsp.v35n2a12

37. Howell AM, Burns EM, Bouras G, Donaldson LJ, Athanasiou T, Darzi A. Can patient safety incident reports be used to compare hospital safety? Results from a quantitative analysis of the english national reporting and learning system data. PLoS One. 2015; 10(12):1-15.

https://doi.org/10.1371/journal.pone.0144107 